\title{
SI physiological units
}

\author{
O. Pellegrino ${ }^{1, \star}$, I. Godinho ${ }^{1}$, C. Oliveira ${ }^{2}$, and E. Filipe ${ }^{1}$ \\ 1 IPQ - Instituto Português da Qualidade, Rua António Gião, 2, 2825-513 Caparica, Portugal \\ 2 IST - Instituto Superior Técnico, Universidade de Lisboa Estrada Nacional 10 (km 139.7), 2695-066 Bobadela LRS, Portugal
}

Received: 1st December 2014 / Accepted: 2nd February 2015

\begin{abstract}
One of the seven base units of the International System of Units (SI), the candela, which is the unit of the luminous intensity, and its derived units are physiological units. The effect of the optical radiation on human beings is taken into account by introducing a weighting factor, the luminous efficiency, to the definition of radiant quantity of interest. Some SI physiological units are frequently used in other metrological domains, such as ionizing radiation, photochemical and biological activities, acoustics and photobiology. This communication is intended to present some principles underlying the definitions of physiological quantities and units associated to these metrological fields.
\end{abstract}

Keywords: Physiological units, luminous intensity, illuminance, ionizing radiation, photochemical units, photobiological units, physiological acoustical units, biological activity of substances

\section{Introduction}

In Portugal, the decree-law No. 128/2010 of December 3 transposed the Directive No. 2009/3/EC of the European Parliament and of the Council of March 11 and adopted SI as the system of legal units of measurement [1]. One of the seven base units, the candela, which is the unit of the luminous intensity, is a physiological unit and so are the units that derive from it, e.g. the luminous flux, $\Phi_{\nu}$, the illuminance, $E_{\nu}$, and the luminance, $L_{\nu}$. The effect of the optical radiation on human beings and the consequent response of the latter are taken into account by introducing a weighting factor into the definition of spectral radiant quantity of interest, $G_{\mathrm{e}}(\lambda)$, leading to the luminous quantity according to:

$$
G_{v}=k_{\mathrm{m}} \int_{380 \mathrm{~nm}}^{760} V(\lambda) d G_{e}(\lambda) .
$$

The weighting factor, $V(\lambda)$, which is called the spectral luminous efficiency, was determined by studying the average spectral response of sets of individuals selectively submitted to reference optical radiation. In equation (1), the factor $k_{\mathrm{m}}$ is the maximum value of the quotient of the luminous flux by the corresponding radiant flux, applied at a monochromatic radiation. For the photopic vision, or daytime vision, i.e. when the normal eye is adapted to levels of luminance greater than ten candelas per square metre, the $k$ maximum value occurs at $\lambda_{\mathrm{m}} \approx 555 \mathrm{~nm}$ and it is equal to $k_{\mathrm{m}}=683 \mathrm{~lm} \mathrm{~W}^{-1}$. For the scotopic vision, or night vision, i.e. when the normal eye is adapted to

\footnotetext{
^ Correspondence: opellegrino@ipq.pt
}

levels of luminance smaller than one thousandth of a candela per square metre, the $k$ maximum value occurs at $\lambda_{\mathrm{m}}=507 \mathrm{~nm}$ and it is equal to $k_{\mathrm{m}}=1700 \mathrm{~lm} \mathrm{~W}^{-1}$ [2].

In this way, radiant flux, radiant intensity, radiance and irradiance with the respective units watt, watt per steradian, watt per square metre per steradian and watt per square metre are associated to the previously mentioned luminous quantities with the respective units lumen, candela, candela per square metre and lux, all of them being physiological units. However, as mentioned in the latest edition of the "SI brochure" [3], some physiological units are aimed at describe biological effects, but with a priori non trivial relationship to SI. Such effects can be found in fields like ionizing radiation, photochemical and biological activities, acoustics and photobiology. In this communication, respective short introductions of these main physiological units are displayed. Two questions are also shortly raised. What is the place of physiologic units in the "new SI" [4] that defines units based on invariants of nature, tendentiously independent of human beings? On the other hand, following the growing interest for the fields of security and health, biometrics and telebiometrics under development ISO/IEC standards 80003 are called "Physiological quantities and their units" [5]. To what extent physiological units and quantities may be involved in?

\section{2 lonizing radiation}

In 2002, the 2nd Recommendation of the International Committee for Weights and Measures (CIPM) settled a relationship to deduce the physiological quantity the dose 
equivalent, $H$, of unit sievert, from the absorbed dose, $D$, of ionizing radiation of unit gray, through a dimensionless quality factor, $Q$, defined as a function of linear energy transfer:

$$
H=Q D \text {. }
$$

Indeed, in 1990, the International Commission on Radiological Protection (ICRP) had introduced protection quantities or physiological quantities, considering weighting factors to be computed (as opposed to operational quantities that are measured) [6].

From the average absorbed dose in $\mathrm{T}$ organ or tissue of the $\mathrm{R}$ radiation, $D_{\mathrm{T}, \mathrm{R}}$, of unit gray and a dimensionless weighting factor of the quality of the $\mathrm{R}$ radiation, $w_{\mathrm{R}}$, the equivalent dose in organ or tissue $\mathrm{T}, H_{\mathrm{T}}$, of unit sievert, was defined by:

$$
H_{\mathrm{T}}=\Sigma_{\mathrm{R}} w_{\mathrm{R}} D_{\mathrm{T}, \mathrm{R}}
$$

The ICRP proposed to use the term radiation weighted absorbed dose in place of equivalent dose aimed at helping to make the quantity more transparent in use. The effective dose, $E$, of unit sievert was defined through a dimensionless weighting factor of $\mathrm{T}$ organ or tissue, $w_{\mathrm{T}}$, according to:

$$
E=\Sigma_{\mathrm{T}} w_{\mathrm{T}} H_{\mathrm{T}}
$$

The $w_{\mathrm{R}}$ and $w_{\mathrm{T}}$ values are released in ICRP publications. The protection quantities that are the organ or tissue equivalent dose and the effective dose are physiological quantities, too.

The quantities of protection are estimated by operational quantities. Among those operational quantities, it can be found the personal dose equivalent $H_{p}(d)$ (for personal monitoring, at an appropriate depth, $d$ ) and ambient dose equivalent $H^{*}(10)$ (for area monitoring, that would be produced at a depth of $10 \mathrm{~mm}$ ).

A dosimeter for absolute measurements is the reference measuring instrument for the operational quantities. Such dosimeters are primary standards and they depend on the quality of the radiation and the medium where the energy is deposited. They may be of ionization chamber (or cavity) type, of in air chamber type or of water or graphite calorimeter type. Currently, EURAMET cooperation projects are aimed at the development of such measuring instruments. Another type of projects [7] is studying a new dosimetric concept considering the growing use of radiotherapy modalities whose biological effect differs from that of conventional high-energy photon beams.

\section{Other physiological units}

\subsection{Photochemical and photobiological units}

For spectral radiant quantities, $G_{\mathrm{e}}(\lambda)$, with SI unit watt per square metre per nanometre, the action spectrum normalized to the maximum, $s_{\mathrm{f}}(\lambda)$, enables to define the dimensionless spectral sensitivity of an $\mathrm{f}$ photochemical or photobiological effect, according to [3]:

$$
G_{f}=\int_{\lambda_{1}}^{\lambda_{2}} s_{\mathrm{f}}(\lambda) d G_{e}(\lambda) .
$$

The example of erythemal effective irradiance $E_{\text {er }}$ is quoted by the "SI brochure" [3]. From a source of ultraviolet radiation, this radiant quantity is obtained by weighting the spectral irradiance of the radiation at wavelength $\lambda$ by the effectiveness of radiation at this wavelength to cause an erythema. In spite of a physiological quantity, no "special status" [3] had been given within the SI for the action spectra and the SI unit for $E_{\text {er remains }}$ W m ${ }^{-2}$.

\subsection{Physiological acoustical unit}

In acoustics domain, the loudness level, $L_{N}$, is a physiological quantity, the unit of which is the phon. Indeed, it corresponds to the perceived sound by the human ear. The loudness level can be obtained from the sound pressure, $L_{p}$, of SI unit pascal, by the following relationship:

$$
L_{N}=L_{p} \eta(\nu, I) .
$$

Here again, a weighting factor is used, $\eta(\nu, I)$, that depends on the sound frequency, $\nu$, and on the sound intensity, $I[8]$, in a non-trivial way. This makes the phon to be a non-SI unit.

\subsection{Unit for biological activity of substances}

Health treatment is a very challenging field for Metrology. Indeed, according to "SI brochure", the mechanism of the specific biological effect that gives some substances their medical use is not yet sufficiently well understood for the biological effect to be quantifiable in terms of physicochemical parameters. This is why units for quantifying the biological activity of these substances cannot yet be defined in terms of SI units.

However, the World Health Organization (WHO) is designated as responsible for defining International Unit (IU) of determined substances, e.g. insulin, vitamin A, vitamin $\mathrm{C}$, vitamin $\mathrm{D}$ and vitamin $\mathrm{E}$, with known biological effects. BIPM projects plan to assign IU to SI value through equivalent mass of such substances of the determined unit $[3,9]$.

\section{Conclusion}

In recent meetings, the Consultative Committee for Units (CCU) has stated that "physiological quantities will become increasingly important in the future" [10]. It followed the very interesting BIPM Workshop on "Physiological Quantities and SI Units" [11], concomitant with the European "Measuring the Impossible" network [12]. This increasing concern of metrology in the field of health is both challenging and important for the progress of human society. 
This communication aimed at highlight the importance of some units that are used as well as units that are needed units in some scientific fields linked with perception by living beings. This concern is not only connected with the field of health, but also with more and better possibilities for living conditions, thanks to available technological and scientific means. It necessarily has to be associated with conceptual progresses in Metrology, through physiological quantities and units within SI.

Due to more and more accurate modelling of the human response to external stimuli, what could be then the role of physiological quantities? For instance, the most efficient software for face recognition are not based on biology models but rather on efficient techniques of calculation like artificial neural [13]. As a matter of fact, the physiological units and quantities in biometrics and telebiometrics ISO/IEC 80003 draft standards "Physiological quantities and their units" [5] are quantities and units among others. Indeed, the documents consider all kinds of physiological interaction between human being and environment through the six modalities: touch, vision, audition, activity, radiation and temperature. The way the interactions are quantified, registered and processed is independent on the perception by human beings.

For some years, there has been a discussion in the metrological community suggested by the BIPM about redefining the current SI base units from invariants of nature [4]. The kilogram, the ampere, the kelvin and the mole would be defined from the Planck constant, the elementary charge, the Boltzmann constant and the Avogadro constant. Within the same logics, the physiological unit, the base unit candela has a corresponding invariant of nature which is an average human eye response to optical radiation. However, this response is expected to vary with time, as is any response to all kinds of physiological units. In the logics of the "new SI", this fact makes question the use of physiological units as base units or the use of concept of base units.

\section{References}

1. Decreto-Lei No. 128/2010 de 3 de dezembro, Diário da República, 1. ${ }^{\mathrm{a}}$ série - No. 234A

2. G. Wyszecki, W.R. Blevin, K.G. Kessler, K.D. Mielenz, Principes régissant la photométrie/Principles governing photometry, BIPM Monographie 83/1, 1983

3. Bureau International des Poids et Mesures, The International System of Units (SI), 8th edition, 2006

4. "New SI", available on: www.bipm.org

5. ISO/WD 80003-2 "Physiological quantities and their units - Part 2: Physics"; IEC/NP 80003-4 "Physiological quantities and their units - Part 4: Biology"

6. ICRP, "1990 Recommendations of the International Commission on Radiological Protection" ICRP Publication 60 Ann. ICRP 21 (1-3), 1991

7. The SIB06 BioQuaRT Consort, available on: https:// www.ptb.de/emrp/bioquart-home.html

8. ISO R 532:1975, Acoustics - Method for calculating loudness level

9. Memorandum of understanding between the World Health Organization (WHO) and the International Committee for Weights and Measures (CIPM) related to traceability of measurements in laboratory medicine, 2002

10. Bureau International des Poids et Mesures, Consultative Committee for Units (CCU) - Report of the 20th meeting (14-16 September 2010) to the International Committee for Weights and Measures, 2010

11. Bureau International des Poids et Mesures, Report on the BIPM Workshop on Physiological Quantities and SI Units, M. Kühne, C. Thomas - Rapport BIPM 2010/05, available on BIPM Website

12. http://minet.wordpress.com/

13. Y. Taigman, M. Yang, M.A. Ranzato, L. Wolf, Deepface: Closing the gap to human-level performance in face verification, Proc. of Computer vision and pattern recognition conference (CVPR, 2014), Columbus, available on: http://www. cvpapers. com/cvpr2014-changelog.html 